Wilfrid Laurier University

Scholars Commons @ Laurier

Physics and Computer Science Faculty

Publications

Physics and Computer Science

$4-1991$

\title{
Time-Explicit Simulation of Wave Interaction in Optical Waveguide Crossings at Large Angles
}

Sai T. Chu

University of Waterloo

Sujeet K. Chaudhuri

University of Waterloo

John W.Y. Lit

Wilfrid Laurier University, jlit@wlu.ca

Follow this and additional works at: https://scholars.wlu.ca/phys_faculty

\section{Recommended Citation}

Chu, Sai T.; Chaudhuri, Sujeet K.; and Lit, John W.Y., "Time-Explicit Simulation of Wave Interaction in Optical Waveguide Crossings at Large Angles" (1991). Physics and Computer Science Faculty Publications. 9.

https://scholars.wlu.ca/phys_faculty/9

This Article is brought to you for free and open access by the Physics and Computer Science at Scholars Commons @ Laurier. It has been accepted for inclusion in Physics and Computer Science Faculty Publications by an authorized administrator of Scholars Commons @ Laurier. For more information, please contact scholarscommons@wlu.ca. 


\title{
Time-explicit simulation of wave interaction in optical waveguide crossings at large angles
}

\author{
Sai T. Chu, Sujeet K. Chaudhuri, and John W. Y. Lit
}

\begin{abstract}
The time-explicit finite-difference time-domain method is used to simulate wave interaction in optical waveguide crossings at large angles. The wave propagation at the intersecting structure is simulated by time stepping the discretized form of the Maxwell's time dependent curl equations. The power distribution characteristics of the intersections are obtained by extracting the guided-mode amplitudes from these simulated total field data. A physical picture of power flow in the intersection is also obtained from the total field solution; this provides insights into the switching behavior and the origin of the radiations.
\end{abstract}

\section{Introduction}

Intersecting waveguides have been proposed as Xswitches ${ }^{1}$ and TE/TM mode splitters. ${ }^{2}$ A number of methods based on the theory of modal or field interference are available in the analysis of these structures. . $^{3-7}$ These methods determine the output power distribution by evaluating the stepwise change of modal or field amplitudes between small sections of the structure. When the reflected or the radiation fields are small, and the coupling between these fields and the guided fields can be neglected, these methods generally provide good results. However, these methods become less applicable as the intersection angle increases and the adiabatic assumption becomes invalid. A rigorous method is the multiple scattering technique ${ }^{8-10}$ which utilizes a multiple interaction picture of coupling between the two waveguides and is formulated in terms of the Green's function for the individual waveguides. The method has been successfully used in the analysis of structures containing small intersection angles and small $\Delta n$.

The purpose of this paper is to study the behavior of the intersecting waveguide at a large angle, typically larger than $10^{\circ}$. Recently, we have applied the finitedifference time-domain (FDTD) method to analyze

John Lit is with Wilfrid Laurier University, Physics Department, Waterloo, Ontario N2L, 3C5, Canada; the other authors are with University of Waterloo, Department of Electrical \& Computer Engineering, Waterloo, Ontario N2L 3G1, Canada.

Received 25 September 1989.

0003-6935/91/121464-07\$05.00/0.

(C) 1991 Optical Society of America. optical guiding structures. ${ }^{11}$ The FDTD method ${ }^{12-15}$ is a very general method that solves the Maxwell's time dependent curl equations numerically. The method simulates wave propagation by time stepping the incident field through the structure. Since it utilizes the total field in the simulation, structures containing large discontinuities can be analyzed. In this paper, the FDTD method is used to investigate how switching takes place at the intersecting waveguide structure at large angles. The simulation is performed for the 2-D step index $\Delta n$ type intersection. The extension to full 3-D simulation is straightforward but requires more extensive computer resources.

Section II briefly describes the FDTD method and the process for determining the power distribution in the individual waveguides from the generated data. Section III presents the simulated switching characteristics of the intersection as a function of angle and width mismatch. Section IV summarizes the results of the paper.

\section{Computation Method}

\section{A. Finite-Difference Time-Domain Method}

The FDTD method is based on the fact that the time derivatives of the $\mathbf{E}$ or $\mathbf{H}$ fields are related to the space derivatives of the $\mathbf{H}$ or $\mathbf{E}$ fields by the Maxwell's time dependent curl equations, so that if the total field distribution over the region of interest is known at a given time, the value of a field component at a later time can be determined.

The time stepping scheme first discretizes the field component function of space and time as

$$
f(x, y, z ; t)=f(i \Delta x, j \Delta y, k \Delta z ; n \Delta t)=f^{n}(i, j, k),
$$


where $i, j, k, n$ are integers $\Delta x, \Delta y$, and $\Delta z$ are space increments, and $\Delta t$ is the time increment. The derivatives in the curl equations are then replaced by their centered difference expressions, so that the field components at $t=n+1$ are expressed as functions of $\mathbf{E}$ and $H$ at $t<n+1$.

For example, the normalized $E_{z}$ equation from the set of time stepping equations is ${ }^{11}$

$$
\begin{aligned}
& E_{z}^{n+1}(i, j, k)=\frac{M_{1}(l)}{M_{2}(l)} E_{z}^{n}(i, j, k) \\
&+\frac{c \Delta t}{\epsilon_{r}(l) M_{2}(l)}\left[\frac{H_{y}^{n+1 / 2}(i+1 / 2, j, k)-H_{y}^{n+1 / 2}(i-1 / 2, j, k)}{\Delta x}\right. \\
&\left.-\frac{H_{x}^{n+1 / 2}(i, j+1 / 2, k)-H_{x}^{n+1 / 2}(i, j-1 / 2, k)}{\Delta y}\right]
\end{aligned}
$$

with

$$
\begin{aligned}
& M_{1}(l)=1-\frac{\sigma(l)}{\epsilon_{r}(l)} \frac{c \Delta t}{2} Z_{0}, \\
& M_{2}(l)=1+\frac{\sigma(l)}{\epsilon_{r}(l)} \frac{c \Delta t}{2} Z_{0},
\end{aligned}
$$

where $l$ denotes a particular material property describing the structure at a given point with conductivity $\sigma(l)$ and relative permittivity $\epsilon_{r}(l), Z_{0}$ is the free space impedance, and $c$ is the velocity of light in a free space.

The simulation starts at $t=0$ with the region of interest containing zero field. For cw excitation, the source distribution with a sinusoidal time variation is fed into the structure at the incident plane. The wave propagation is simulated by repeatedly applying the time stepping equation (2) and the associated equations for $E_{x}, E_{y}, H_{x}, H_{y}$, and $H_{z}$. The simulation is completed when the steady state is observed at the output of the structure. The solution gives the total field distribution over the region at the discretized space location and time instant.

Readers are referred to Refs. 11 and 12 and other references in Ref. 12 for the implementation details of FDTD. Some of the important issues for the implementation are error and stability analysis, source generation, and truncation boundary conditions. The FDTD simulator has been validated extensively in solving optical waveguide problems, in particular it has been validated against the exact solution for the case of parallel slab couplers ${ }^{11}$ and against the rigorous solutions for the case of an abrupt step junction. ${ }^{16}$

\section{B. Single $\Delta n$ Type Waveguide Intersection}

Figure 1 shows the geometry of the two intersecting waveguides. The guides are single mode step index waveguides, except in the width mismatch case, where guide 2 may support two modes. In this configuration, guides 1 and 2 are referred to as the bar and cross channels of the intersecting structure, so that at the bar or cross state, a major part of the output is distributed in the bar or cross channel. At the incident plane $z=z_{\text {inc }}$, the fundamental $\mathrm{TE}_{0}$ or $\mathrm{TM}_{0}$ mode of the slab waveguide is introduced into the structure through

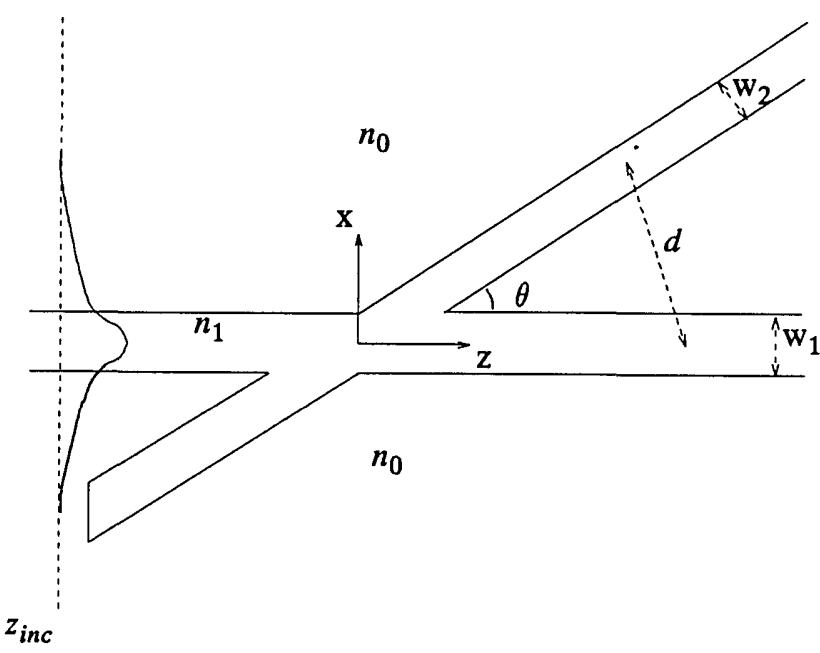

Fig. 1. Schematic diagram of two intersecting step index waveguides. Waveguide 2 is truncated so that waveguide 1 will support the fundamental $\mathrm{TE}_{0}$ and $\mathrm{TM}_{0}$ mode of a single guide at $z=z_{\text {inc. }}$

waveguide 1 . The separation between the two guides at $z_{\text {inc }}$ is so chosen that $<0.1 \%$ of the incident power is launched into the end of guide 2 to ensure that only side coupling occurs between the waveguides. The propagation of the incident field and the wave interaction at the intersection is simulated by the FDTD method. Simulation is completed when the steady state is observed at the output of the waveguides. At the steady state, the field observed at a point in the output channels will have the same time variation as the source.

As stated before, the result from the simulation is in the total field form. The field distribution at the output of the waveguides is a combination of the guided and the radiation modes of the individual waveguides and the scattered field generated by the intersection. If we allow the minimum separation of the waveguide centers $d$ to extend to infinity, where only the guided mode will remain in the waveguides, one can determine the power in the individual waveguide directly from the field amplitude. However, in practice it is necessary to truncate the infinite structure, and the output field from the truncated geometry will contain the radiation and scattered fields in addition to the guided modes.

To evaluate the guided power in the individual guides, we employ a mode extraction technique ${ }^{16}$ that uses the least-squares approach to extract the guided mode amplitude from the total field distribution. The FDTD generated total field distribution along a plane perpendicular to the $z$-axis is compared with the guided mode distribution; the amplitudes of the guided modes are adjusted until the square difference of the two distributions is minimized. Figure 2 shows the variation of the guided $\mathrm{TE}_{0}$ mode amplitude as a function of waveguide separation $d$; it shows the convergence of the modal amplitudes with increasing $d$. The variation of the extracted amplitudes at small $d$ is 


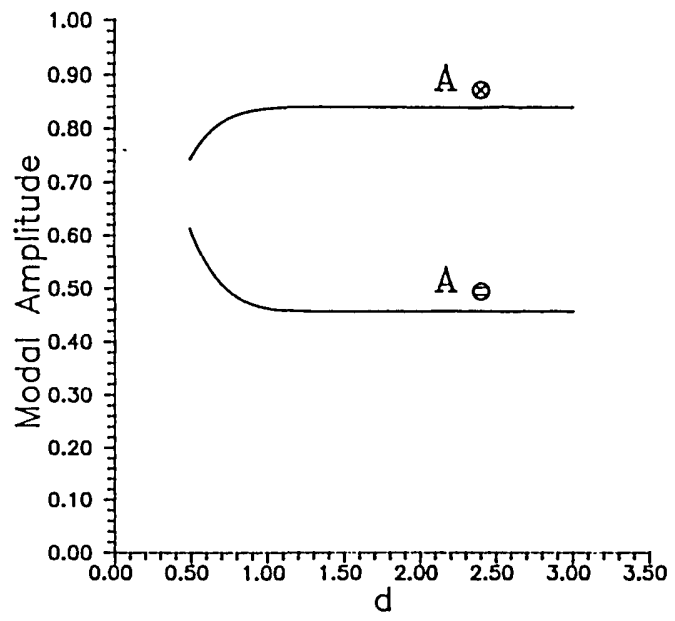

Fig. 2. $\mathrm{TE}_{0}$ amplitude vs waveguide separation $d$ with $n_{0}=2.30$, $w_{1}=w_{2}=0.5 \mu \mathrm{m}, \lambda=1.30 \mu \mathrm{m}, \theta=14^{\circ}$, and $V=2.93$.

because the coupling between these guided modes is still taking place in this region. As $d$ increases, the overlap of the guided modes in the two guides becomes small, and one can treat the geometry as two isolated waveguides. Furthermore, the superposition of the fundamental modes of the individual guides cannot represent the normal mode distribution of the fivelayer structure, so that the extracted modal amplitudes depend on the amount of field overlap between the fundamental modes and the local normal modes. The optical length of the intersection, within which coupling between the waveguides occurs, can be measured from Fig. 2. As observed in Fig. 2, the amplitudes of the guided modes become stable when $d>1.0$ $\mu \mathrm{m}$; hence the optical length of the structure is the distance between the planes where $d=1.0 \mu \mathrm{m}$. In the results in the next section the modal coefficients will be extracted from the FDTD generated data at $d=1.5$ $\mu \mathrm{m}$, where there is very little coupling between the fields in the two output guides.

\section{Simulation Results and Discussion}

The switching characteristics of two intersecting identical waveguides with respect to the intersection angle are shown in Fig. 3. The geometries discussed have $n_{0}=2.30, w_{1}=w_{2}=0.5 \mu \mathrm{m}, \lambda=1.30 \mu \mathrm{m}$, and mode confinement parameter

$$
V=\frac{2 \pi}{\lambda} w_{1} \sqrt{n_{1}^{2}-n_{0}^{2}}=2.93 \text {. }
$$

The material considered in this paper is assumed to be isotropic, although anisotropic material can be modeled by simply assigning different values for $n_{x}$ and $n_{z}$. Here we only consider the variation of switching characteristics due to changes in the geometry. The discretizations used in these simulations are $\Delta x=\Delta z=$ $2 c \Delta t=\lambda / 26$. With the size of the computational region depending on the value of $\theta$, the number of cells used ranges from 20 to 60 thousands, and they require 1 to 3 thousands time steps for the simulations to reach steady state, which corresponds to 2-15 CPU min on

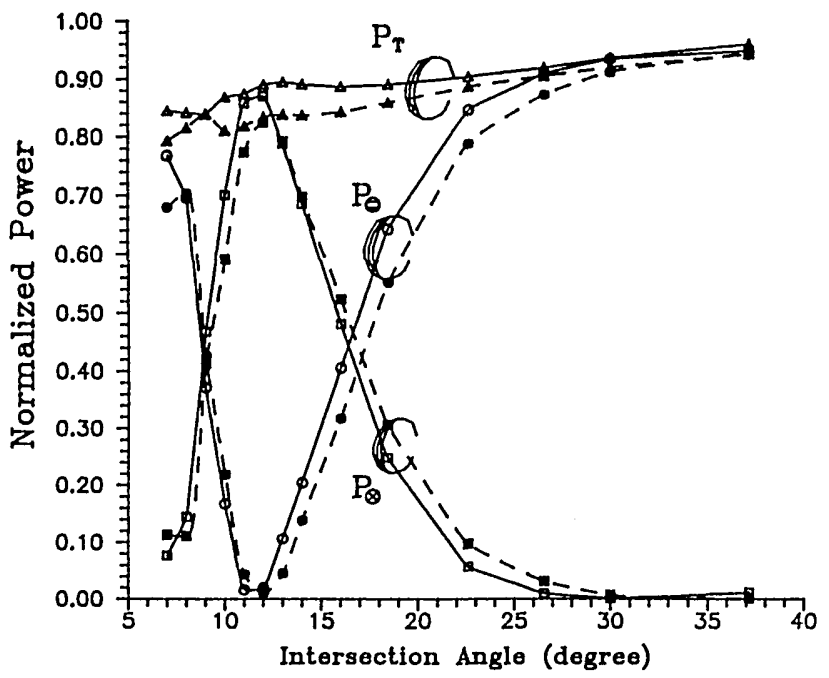

Fig. 3. Switching characteristics as a function of intersection angle $\theta$ with $d=1.5 \mu \mathrm{m}$. Waveguide parameters are the same as in Fig. 2 (TE, solid line; TM, dashed line).

the MIPS/2000 computer. We have employed the second-order boundary condition ${ }^{17}$ in these simulations.

As shown in Fig. 3, the switching characteristics with respect to the intersection angle for the TE and the TM cases differ only slightly, indicating that the crossing is not very sensitive to polarization. However, the losses are found to be larger in the TM cases as the TM modes have smaller propagation constants and they are more susceptible to radiation. For example, at $\theta=$ $11^{\circ}$, the power in the bar channel is about the same in either polarizations; however, the cross channel for the TM case contains less power. At larger angles, $\theta>20^{\circ}$, the excess loss decreases monotonically with increasing angle. The bar channel continues to gain power. For $\theta>20^{\circ}$ it is not possible to obtain the cross-state. When $\theta<20^{\circ}$, the excess loss behavior is slightly different. Although the loss still increases with decreasing angle, this increase is not monotonic. The loss appears to have localized maximum and minimum. This behavior of the loss is also reported in Ref. 10.

Figure 4 shows the time-averaged Poynting vector diagrams of the TE waves for two angles. The steady state values of the field components are first determined from successive time iterations. The time-averaged Poynting vector $\mathbf{S}(t)=\mathbf{E}(t) \times \mathbf{H}(t)$ is then calculated at each computation cell. The lengths of the arrows in the diagram are scaled linearly according to their power magnitudes, and the contour levels correspond to the maximum power magnitude in the region.

The power flow through the intersection at $\theta=7$ and $11^{\circ}$ is shown in Figs. 4(a) and (b). In Fig. 4(a), we observe that when the separation between the two waveguides becomes small, the power in the bar channel begins to couple into the cross channel. Strong coupling is found at the common section of the two 


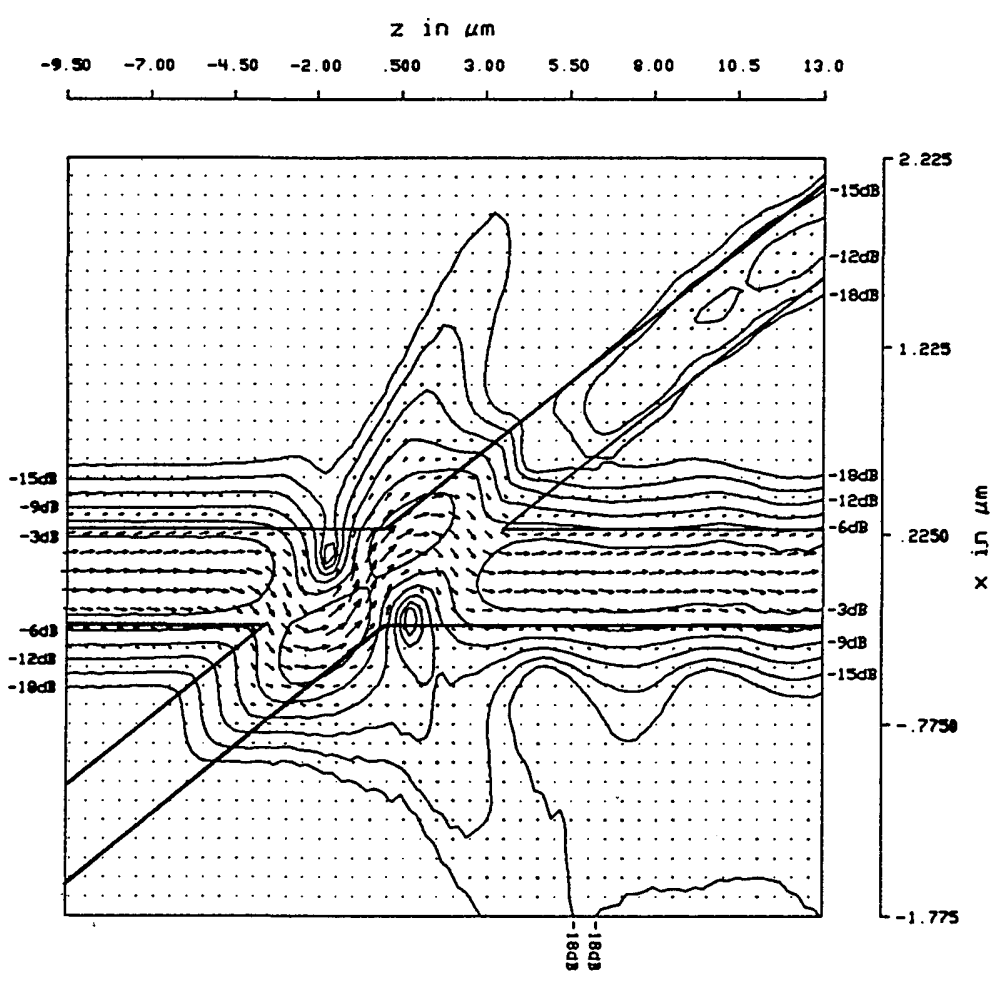

(a)

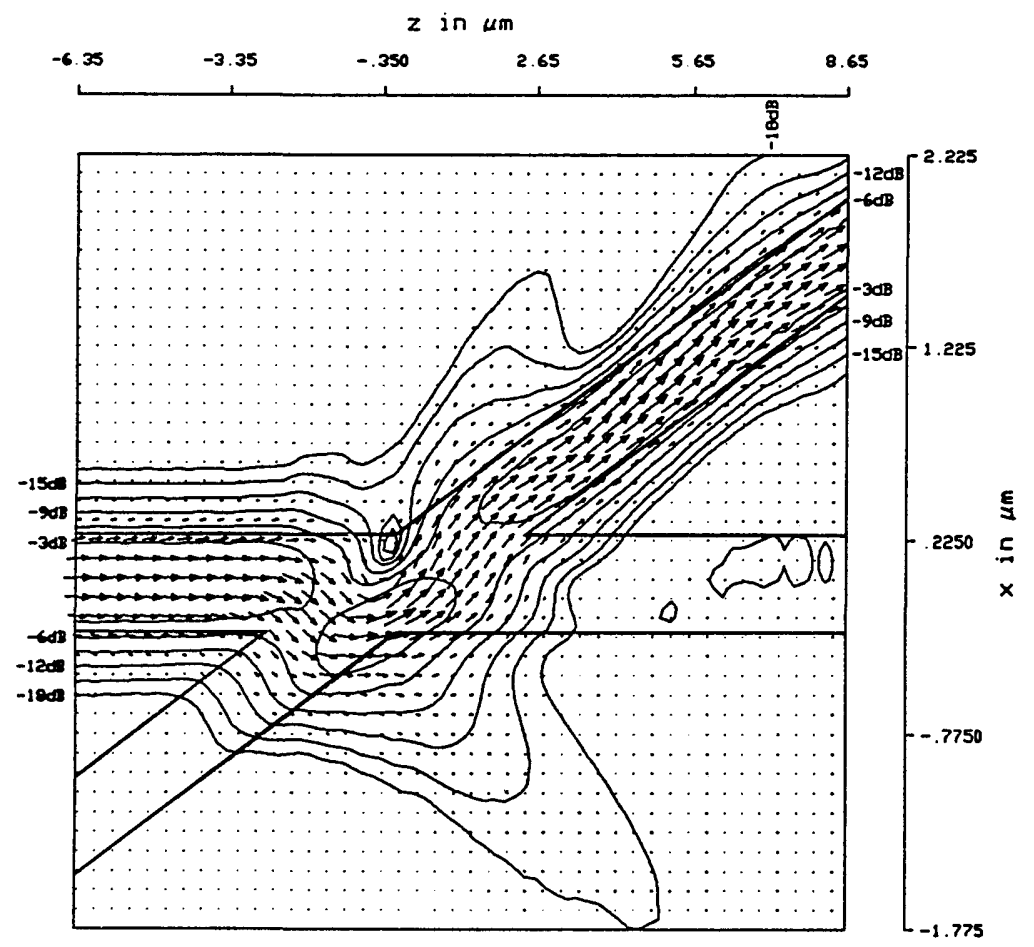

(b)
Fig. 4. (a) Poynting vector diagram at $\theta=7^{\circ}$. Parameters are the same as in Fig. 2. (b) Poynting vector diagram at $\theta=11^{\circ}$. Parameters are the same as in Fig. 2. waveguides, where the interference of the incoming guided mode and the excited higher-order modes causes the power to flow upward and then downward. The total number of up-down oscillations is related to the physical and the optical lengths of the common section. An increase in either lengths will increase the number of oscillations. The physical length of the section can be increased by reducing the intersection angle or increasing the waveguide widths. The optical length is controlled by the mode confinement number $V$, which can be increased by reducing the wavelength or increasing the index difference $\Delta n$. For a large 


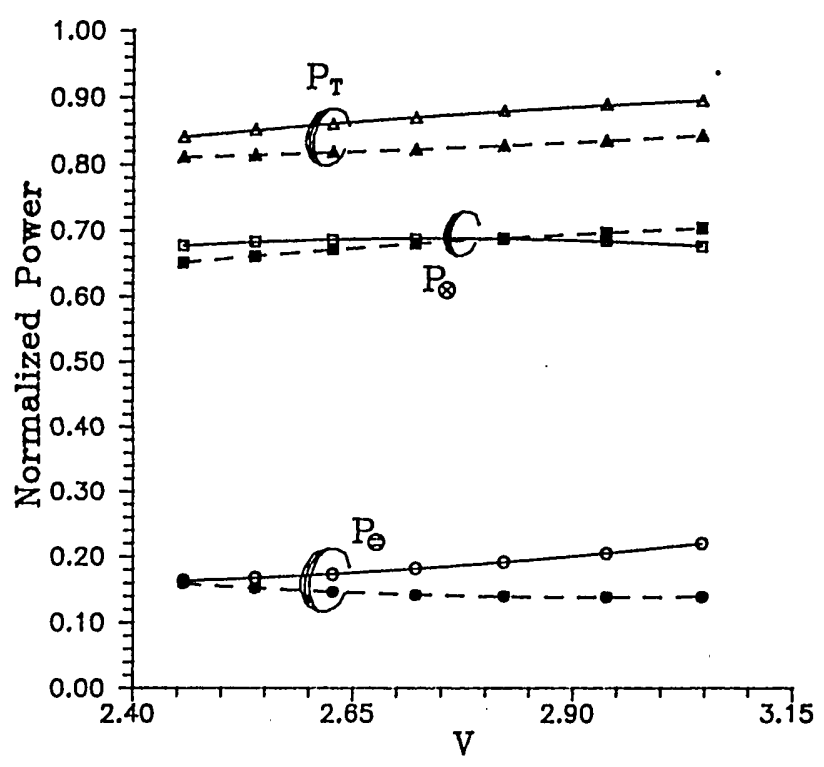

Fig. 5. Switching characteristics as a function of $V$ with $\theta=14^{\circ}$. Waveguide parameters are $n_{0}=2.30, w_{1}=w_{2}=0.5 \mu \mathrm{m}, \lambda=1.25-1.55$ $\mu \mathrm{m}$, and $d=1.5 \mu \mathrm{m}$ (TE, solid line; TM, dashed line).

intersection angle, where the physical length is small, one must increase the optical length by increasing $\Delta n$ to obtain a cross-state. The distribution of the output power is found to be strongly dependent on the behavior of the power path at the output of the common section. If the path is in the direction of the bar channel, as in Fig. 4(a), the major portion of the output power will be in the bar channel. Similarly, the power path in Fig. 4(b) is in the direction of the cross channel, and the intersection is in the cross-state. Using the Poynting vector diagram one can adjust the output angle to obtain the required switching characteristics.

It is observed that the radiation is generated by the bends of the power path; power is radiated away from the intersection at bends. There are two main radiation loops found in all cases, with the upward radiation loop radiating in a direction closer to the direction of the cross channel. However, the downward radiation loop and the bar channel are separated by a larger angle. As a result the coupling appears to be stronger between the upward radiation loop and the cross channel. In Fig. 4(a), one can see that there is no observable direct power flowing into the cross channel, but the cross channel has picked up power away from the common section. The excess loss of the structure depends on the amount of generated radiation and the amount of coupling between the guided modes and the radiation fields. An increase in the number of oscillations will increase the amount of radiation. This causes the loss to increase with decreasing angle, as shown in Fig. 3. It should be mentioned that although the magnitude is small, the Poynting vectors in the upper and lower left corners of Fig. 4 are pointed in the negative $z$-direction, showing the reflected power from the intersection.

The variation of the power distribution with respect

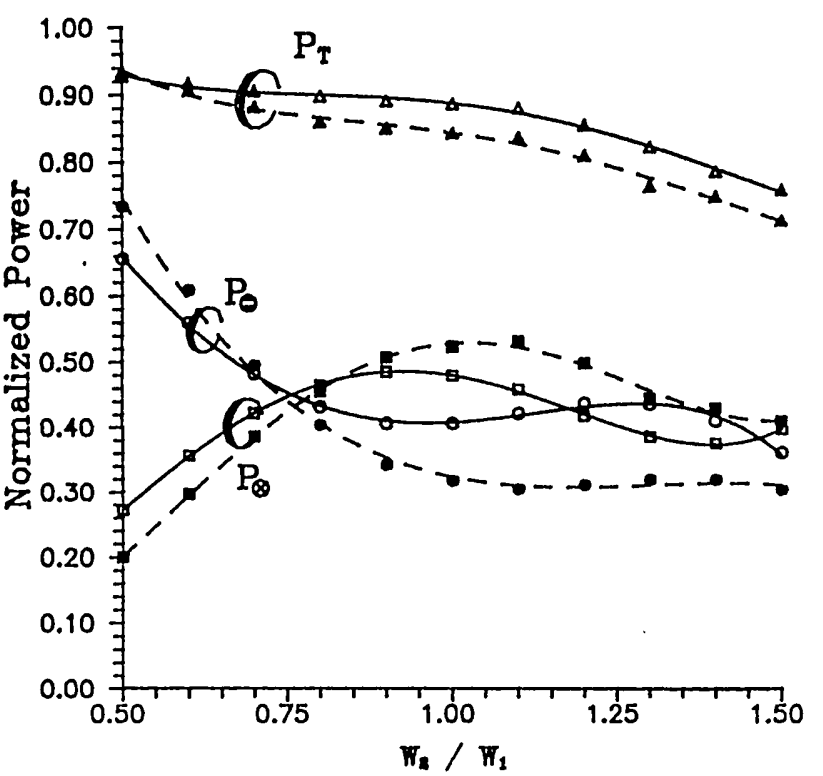

Fig. 6. Switching characteristics as a function of $w_{2} / w_{1}$ with $\theta=16^{\circ}$. Waveguide parameters are $n_{0}=2.30, \lambda=1.30 \mu \mathrm{m}, w_{1}=0.5 \mu \mathrm{m}$, $V_{1}=2.93$, and $d=1.5 \mu \mathrm{m}$ (TE, solid line; TM, dashed line).

to $V$ is shown in Fig. 5. The parameters used are the same as in Fig. 2 with $\theta=14^{\circ}$. The higher loss value at smaller $V$ is because of the weaker power confinement, which causes more power to radiate from the intersection at the bends. The switching behavior is more difficult to interpret. It appears that the switching characteristics for the two polarizations behave differently with $V$. As $V$ increases, in the TE case, the power levels in the two channels approach each other. In the TM case, their difference increases. This results in a large crosstalk difference between the two polarizations at large $V$.

The switching characteristics calculated for different width mismatches $w_{2} / w_{1}$ are shown in Fig. 6. It shows that the loss increases with increasing $w_{2}$. This effect can be observed from the Poynting vector diagrams in Fig. 7. At small $w_{2}$, the small crossing region causes a smooth power-path bend, which releases only a small amount of radiation. At large $w_{2}$, the power path is more sharply bent and more radiation results. Note that in Fig. 7(b) the cross channel supports two modes. The oscillatory pattern of the power path in the cross channel is due to the beating of the two guided modes. This pattern will continue down the channel. However, the oscillatory behavior in the bar channel is due to the interference of the guided mode with the radiation modes. As the amplitudes of these radiation modes decrease along the channel, the power path will become parallel to the channel.

\section{Conclusions}

We have simulated the wave propagation in waveguides intersecting at large angles, using the FDTD method. The analysis of structures containing large discontinuities is possible, because the FDTD method utilizes the total field in its simulation. The complete 


\begin{tabular}{|c|c|c|c|c|c|}
\hline \multicolumn{6}{|c|}{$z$ in $\mu m$} \\
\hline-4.25 & -2.25 & -.250 & 1.75 & 3.75 & 5.75 \\
\hline
\end{tabular}

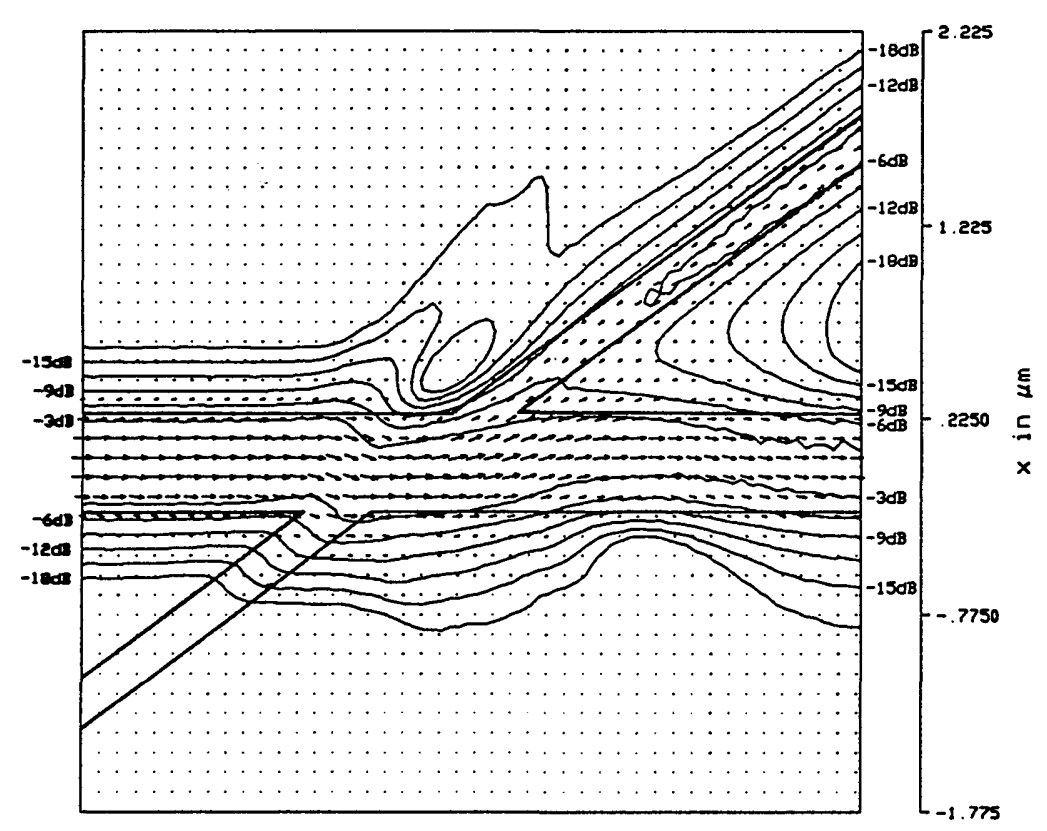

(a)

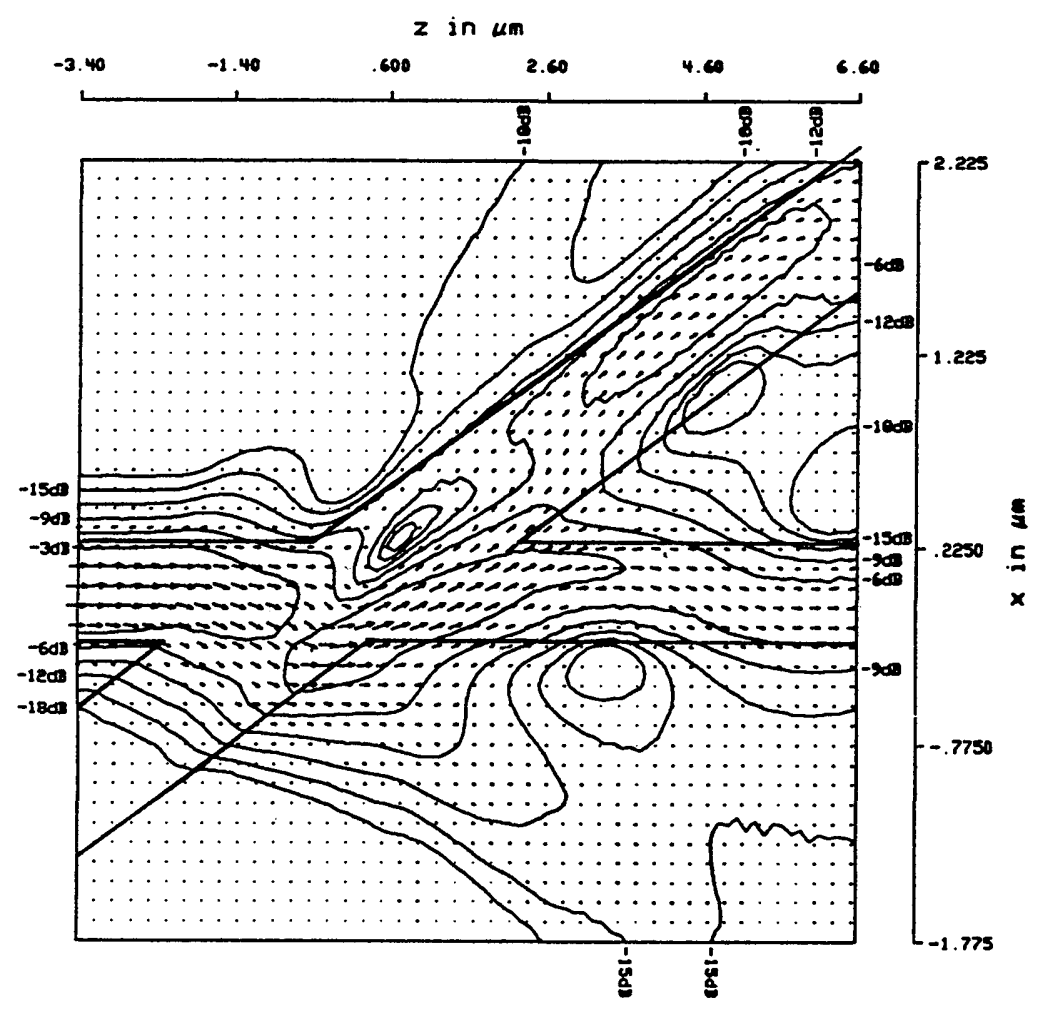

(b)

field solution obtained from the simulation is used to generate the Poynting vector diagram, which shows the direction of the power flow through the intersection. The output distributions of the intersecting waveguides depend on the direction of the power flow at the common section of the waveguides. The radia-
Fig. 7 (a) Power flow through the intersection with $w_{2} / w_{1}=0.5$. Parameters are the same as in Fig. 6. (b) Power flow through the intersection with $w_{2} / w_{1}=1.5$. Parameters are the same as in Fig. 6. tion is observed to originate at the power-path bends. Thus the amount of radiation generated at the intersection is a function of the total number of bends and the sharpness of the bends. Although the FDTD algorithm is a computer intensive scheme and the size of the problem that can be solved is dependent on the 
computer resource, the finite-difference scheme takes full advantage of a modern computer that has vector and parallel processing capabilities. Finally, the FDTD approach gives physical insight into the wave propagation through complex structures; this will enhance the design of new optical components.

\section{References}

1. A. Neyer, "Electro-Optic X-Switch Using Single-Mode Ti:LiNbO ${ }_{3}$ Channel Waveguides," Electron Lett. 19, 553-554 (1983).

2. H. Nakajima, T. Horomatsu, M. Seino, and I. Sawaki, "Crosstalk Characteristics of $\mathrm{Ti}: \mathrm{LiNbO}_{3}$ Intersecting Waveguides and their Application as TE/TM Splitters," IEEE Trans. Microwave Theory Tech. MTT-30, 617-621 (1982).

3. G. E. Betts and W. S. C. Chang, "Crossing-Channel Waveguide Electrooptic Modulators," IEEE J. Quantum Electron. QE-22, 1027-1038 (1986).

4. K. Goel and W. S. C. Chang, "Extinction Ratio Degradation due to Asymmetry in Zero-Gap Directional Coupling and Crossing Channel Switches," IEEE J. Quantum Electron. QE-23, 22162223 (1987).

5. A. J. Weierholt, S. Neegard, and A. R. Mickelson, "Eigenmode Analysis of Optical Switched in $\mathrm{LiNbO}_{3}$-Theory and Experiments," IEEE J. Quantum Electron. QE-24, 2477-2489 (1988).

6. J. P. Weber, L. Thylen, and S. Wang, "Crosstalk and Switching Characteristics in Directional Couplers," IEEE J. Quantum Electron. QE-24, 537-548 (1988).

7. A. Neyer, W. Mevenkamp, L. Thylen, and B. Lagerstrom, “A Beam Propagation Method Analysis of Active and Passive Waveguide Crossings," IEEE/OSA J. Lightwave Technol. LT-3, 635-642 (1985).
8. N. Agrawal, L. McCaughan, and S. R. Seshadri, "A Multiple Scattering Interaction Analysis of Intersecting Waveguides," J. Appl. Phys. 62, 2187-2193 (1987).

9. N. Agrawal and L. McCaughan, "Radiation Losses in Intersecting Optical Waveguides," J. Appl. Phys. 65, 4509-4516 (1989).

10. L. McCaughan, N. Agrawal, and G. A. Bogert, "Novel Physical Effects in Intersecting Waveguides," Appl. Phys. Lett. 51, 13891391 (1989).

11. S.-T. Chu and S. K. Chaudhuri, "Finite-Difference Time-Domain Method for the Design and Analysis of Guided-Wave Optical Structures," IEEE/OSA J. Lightwave Technol. JLT-7, 2033-2038 (1989).

12. A. Taflove and K. R. Umashankar, "Finite-Difference TimeDomain (FD-TD) Modeling of Electromagnetic Wave Scattering and Interaction Problems," IEEE AP-S Newsletter 5-20 (Apr. 1988).

13. V. Shanka, W. Hall, and A. Mohammadian, "A CFD-Based Finite-Volume Procedure for Computational Electromagnetics-Interdisciplinary Applications of CFD Methods," in AIAA Ninth Computational Fluid Dynamics Conference, Buffalo (June 1989), paper AIAA-89-1987.

14. K. S. Yee, "Numerical Solution of Initial Boundary Value Problems Involving Maxwell's Equations in Isotropic Media," IEEE Trans. Antennas Propag. AP-14, 302-307 (1966).

15. R. Holland, "Threde: A Free-Field EMP Coupling and Scattering Code," IEEE Trans. Nucl. Sci. NS-24, 2416-2421 (1977).

16. S. T. Chu and S. K. Chaudhuri, "Combining Modal Analysis and the FDTD Method in the Study of Dielectric Waveguide Problems," IEEE Trans. Microwave Theory Tech. MTT-38, 17551760 (1990).

17. G. Mur, "Absorbing Boundary Conditions for the Finite-Difference Approximation of the Time-Domain Electromagnetic Field Equations," IEEE Trans. Electromagn. Compat. EMC23, 707-725 (1980). 